\title{
Open Source Software Library for Thermo-Hydro-Mechanical Coupled Processes in Python
}

\author{
Carsten Rücker \\ Federal Office for the Safety of Nuclear Waste Management (BASE), Berlin, Germany \\ Correspondence: Carsten Rücker (carsten.ruecker@bfe.bund.de) \\ Published: 10 November 2021
}

\begin{abstract}
This contributed poster shows the current state of development of a finite element implementation as part of an open source software library (OSSL) for the simulation of thermo-hydro-mechanical (THM) coupled processes. The reliable handling of numerical methods is fundamental for the understanding of scientific interrelationships and thus, a crucial prerequisite for modeling THM scenarios, as well as for the understanding and evaluation of preliminary safety investigations during the site selection process for the storage of high-level radioactive waste. There are several motivations for developing an in-house OSSL, which will allow us to:

- Build capacity and maintenance within BASE (Federal Office for the Safety of Nuclear Waste Management) regarding issues of the numerical modeling of safety-relevant aspects on the long-term safety analyses specified by the German legislator in the site selection process.

- Develop a collection of known benchmarks and evaluation examples for the comparison of different software tools, applying a uniform interface to simplify the use of the available highly specialized open source codes.

- Diversify the testing possibilities regarding the preliminary safety investigations by means of our own, independent modeling software.
\end{abstract}

- Document basic THM scenarios for internal or, if necessary, public technical training, e.g., density-driven fluid flow (Fig. 1), convergence in salt, temperature propagation in the repository area, crack development, diffusive or advective mass transport.

- Ensure transparency and, in principle, might allow for appropriately proven-quality (validated) and documented simulation tools for the public regarding questions about the preliminary safety investigations during the site selection process.

The development of the OSSL is mainly based on the scripting language Python, which allows the necessary flexibility for the diverse fields of application and at the same time enables maximum transparency for all aspects of the source code. To ensure the high quality of the software, state of the art development tools are used (e.g., version control, automated tests, and documentation generation).

Figure 1 shows our preliminary simulation results of the so-called Elder problem (Elder, 1967), a popular standard benchmark for thermo-hydrogeological coupling in which fluid motion in a porous medium is driven by buoyancy forces.

Kurzfassung. Das vorgestellte Poster zeigt den Entwicklungsstand einer inhouse entwickelten Open-SourceImplementierung der Finite-Elemente-Methode als Teil einer Softwarebibliothek zur Simulation thermischhydromechanisch (THM) gekoppelter Prozesse. Der sichere Umgang mit numerischen Methoden ist eine Grundlage für das Verständnis naturwissenschaftlicher Zusammenhänge und damit eine Voraussetzung zur modellhaften Abbildung von THM-Szenarien sowie zum Verständnis und zur Bewertung von vorläufigen Sicherheitsuntersuchungen im Auswahlverfahren für einen Standort für hochradioaktive Abfälle. Für die Entwicklung einer eigenen Open-Source-Softwarebibliothek gibt es verschiedene Beweggründe: 
- Förderung des Kompetenzerhalts und -aufbaus innerhalb des BASE (Bundesamt für die Sicherheit der nuklearen Entsorgung) zu Fragen der numerischen Modellierung sicherheitsrelevanter Aspekte in Hinblick auf die vom Gesetzgeber festgelegten Langzeitsicherheitsanalysen im Standortauswahlverfahren.

- Aufbau einer Sammlung von bekannten Benchmarks und Evaluationsbeispielen zum Vergleich verschiedener Softwarelösungen verbunden mit der Erstellung einer einheitlichen Anwenderschnittstelle zu alternativ verfügbaren Open-Source-Speziallösungen.

- Diversifizierung der Prüfmöglichkeiten bezüglich der vorläufigen Sicherheitsuntersuchungen durch eine eigene, unabhängige Modellierungssoftware.

- Aufarbeitung und Dokumentation von grundlegenden THM-Szenarien für interne oder ggf. auch öffentliche fachliche Weiterbildung; beispielsweise dichtegetriebene Fluidbewegung (Abb. 1), Konvergenz im Salz, Temperaturausbreitung im Endlagerbereich, Rissentwicklung, diffusiver oder advektiver Stofftransport.

- Die Wahl eines Open-Source-Ansatzes gewährleistet Transparenz und erlaubt prinzipiell auch eine Bereitstellung von entsprechend qualitätsgesicherten und dokumentierten Simulationswerkzeugen zu Fragen der vorläufigen Sicherheitsuntersuchungen bei der Standortauswahl für die Öffentlichkeit.

Die Entwicklung der Programmbibliothek erfolgt in wesentlichen Teilen in der Skriptsprache Python, was auf der einen Seite die nötige Flexibilität für die vielfältigen Einsatzgebiete der Software erlaubt und gleichzeitig maximale Transparenz für alle Aspekte des Quellcodes ermöglicht. Zur Gewährleistung einer hohen Qualität der Software kommen zeitgemäße Werkzeuge der Softwareentwicklung zum Einsatz (beispielsweise Versionskontrolle, automatisierte Tests und Dokumentationserzeugung).

Als Beispiel zeigt Abb. 1 das Ergebnis der Simulation einer dichtegetriebenen Fluidbewegung im porösen Medium nach dem sog. Elder-Problem (Elder, 1967), einem beliebten Standardbenchmark zur thermischhydrogeologischen Kopplung.

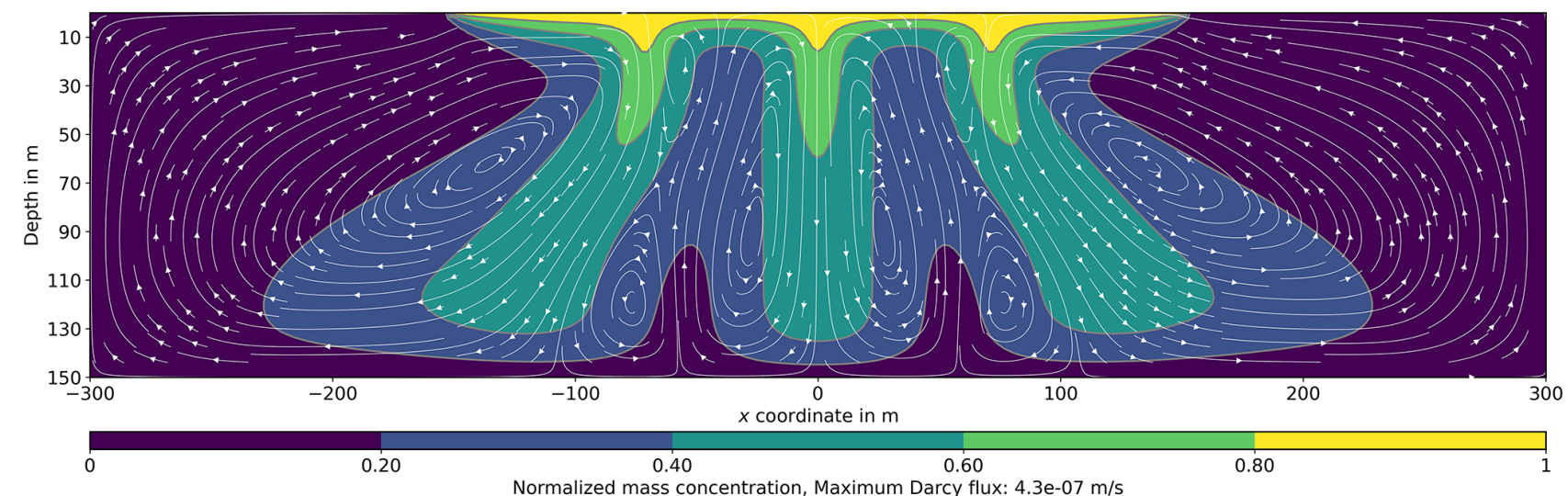

Figure 1. Simulation result of density-driven fluid flow in porous medium (Elder problem): normalized mass concentration (colored patterns) and Darcy flux field (white vectors).

\section{References}

Elder, J. W.: Transient convection in a porous medium, J. Fluid Mech., 27, 609-623, https://doi.org/10.1017/ S0022112067000576, 1967. 